\title{
Cooperative response and clustering: Consequences of membrane-mediated interactions among mechanosensitive channels
}

\author{
Lucas D. Fernandes,,${ }^{1, *}$ Ksenia Guseva,,${ }^{2, \dagger}$ and Alessandro P. S. de Moura ${ }^{3, \ddagger}$ \\ ${ }^{1}$ Departamento de Entomologia e Acarologia, Escola Superior de Agricultura Luiz de Queiroz - Universidade de São Paulo (USP), \\ 13418-900, Piracicaba, São Paulo, Brazil \\ ${ }^{2}$ Theoretical Physics/Complex Systems, ICBM, University of Oldenburg, 26129 Oldenburg, Germany \\ ${ }^{3}$ Institute for Complex Systems and Mathematical Biology, King's College, University of Aberdeen, AB24 3UE, Aberdeen, United Kingdom
}

(Received 19 May 2017; published 21 August 2017)

\begin{abstract}
Mechanosensitive channels are ion channels which act as cells' safety valves, opening when the osmotic pressure becomes too high and making cells avoid damage by releasing ions. They are found on the cellular membrane of a large number of organisms. They interact with each other by means of deformations they induce in the membrane. We show that collective dynamics arising from the interchannel interactions lead to first- and second-order phase transitions in the fraction of open channels in equilibrium relating to the formation of channel clusters. We show that this results in a considerable delay of the response of cells to osmotic shocks, and to an extreme cell-to-cell stochastic variations in their response times, despite the large numbers of channels present in each cell. We discuss how our results are relevant for E. coli.
\end{abstract}

DOI: 10.1103/PhysRevE.96.022410

\section{INTRODUCTION}

Abrupt changes in the osmolarity of the environment is a hazard most organisms are subject to at one time or another [1-6]. A sudden drop in osmolarity (an osmotic shock) will cause water to rush into a living cell and requires an immediate response by the cell to prevent it from getting damaged or undergoing lysis from the increased tension on the cellular membrane. Mechanosensitive (MS) channels are ion channels located on the cell membrane, which open when the membrane tension becomes too high $[7,8]$, and play a crucial role in the cell's defence mechanism against osmotic shocks $[9,10]$. They act as safety valves, releasing ions and decreasing the osmotic pressure and the membrane tension. MS channels are found in many organisms and have been well characterized in the bacterium E. coli [11-13].

The cellular membrane in which the MS channels are inserted is a lipid bilayer. The interior of the bilayer is hydrophobic, making it energetically favourable for it to thicken or compress to match the hydrophobic parts of the channel proteins inserted in the membrane [14]. This results in a deformation profile around each channel, with the thickness of the bilayer being a function of position. This deformation mediates a short-range effective force between two neighboring channels, similar to the force between two nearby corks floating on water, which interact through the deformation they induce on the surface of water. This interaction can be attractive or repulsive, depending on the shapes of the two molecules. Furthermore, a theoretical analysis suggests that the interaction between two neighboring channels lowers the tension needed to open them during an osmotic shock [15], raising the possibility that their function could be influenced by their spatial distribution on the membrane (as already noticed for other membrane proteins $[16,17])$. This is reinforced by

\footnotetext{
*1dfernandes@usp.br

${ }^{\dagger}$ ksenia.guseva@uni-oldenburg.de

$\ddagger$ a.moura@abdn.ac.uk
}

the fact that the channels' attractive forces suggest that they may agglomerate into clusters. Our goal in this paper is to determine the consequences that the interchannel interaction has on the dynamics of this system, focusing in particular on channel clustering and its consequences for the cell's response to osmotic shocks.

A preliminary study of mechanosensitive channel clustering was done in Refs. [18,19]. In that work, diffusion, leading to the formation of clusters of channels, and opening were considered two separate processes. This assumption made the model easier to analyze, but it is hard to justify: in reality, diffusion and gating take place simultaneously.

In this work, we formulate a model of the collective dynamics of MS channels, where diffusion and gating are considered simultaneous, and no assumption of time separation between clustering and gating is made. Using a combination of analytic techniques and numerical simulations, we analyze the equilibrium and the dynamics of the system, focusing in particular on the response of the channels to osmotic shocks. We find that the interplay between the spatial and the internal degrees of freedom of the channels leads to unexpected collective phenomena, with possible implications for their biological function. We show that the fraction of open channels undergoes a phase transition as the membrane tension increases; and this transition changes from second-order to first-order as the density of channels crosses a critical value. We explain this change in the nature of the transition as the result of collective gating induced by a cluster of channels which appears for high densities. Studying the time evolution of the system after applying an osmotic shock, we find that clustering leads to dramatic changes in the channels' response, slowing considerably their gating. In addition, clustered channels show extreme ensemble variations in their response times, despite the large numbers of channels present in each cell, which could translate into large stochastic cell-to-cell differences in response times in a population of cells. Finally, we discuss how our results are relevant for the stress response of $E$. coli and other organisms. 


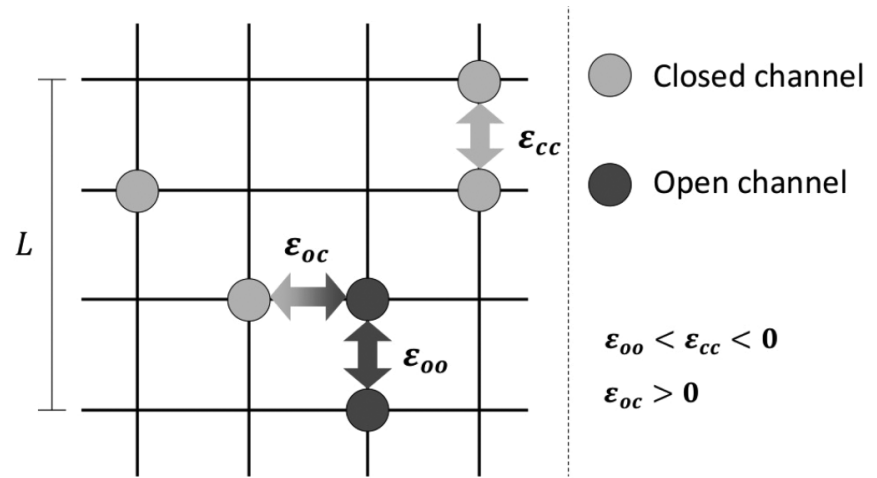

FIG. 1. Diagram representing the lattice model. Channels interact with nearest neighbors with energies $\varepsilon_{o o}, \varepsilon_{c c}$, and $\varepsilon_{o c}$, depending on the states of the interacting channels.

\section{MODEL}

In our model, we regard the cellular membrane as a two-dimensional (2D) square lattice of size $L$, where each of the sites can be either empty or occupied by a channel. We focus on the mechanosensitive channels of large conductance (MscL), which have been well studied in this context [20-22]. The total number $N$ of channels is regarded as constant, so that the density $\rho$ of channels (mean number of channels per lattice site) is fixed; we are in the canonical ensemble. We consider that MscL can be in one of two states, closed or open. The interaction energies between two channels have been obtained by minimizing the energy functional defined by the deformation profile [15]. For small distances (a few nanometers) between channels, if $\varepsilon_{o o}, \varepsilon_{c c}$, and $\varepsilon_{o c}$ are the interaction energies between a pair of open channels, a pair of closed channels, and an open and a closed channel, respectively, we have $\varepsilon_{o o}<\varepsilon_{c c}<0$ and $\varepsilon_{o c}>0$ : two open channels and two closed channels attract each other, the attraction being stronger in the first case; and a closed channel repels an open channel (see Ref. [18] for the complete energy profiles). Figure 1 illustrates our lattice model.

If we consider that all channels are in the same state, without the possibility of gating, this model is exactly the lattice gas model. The 2D lattice gas model is exactly solvable, due to its equivalence to the Ising model [23], and presents a phase transition from a homogeneous to a clustered channel distribution as the density of channels increases [18].

The model we describe shares some similarities with the spin-1 Ising model analyzed in a mean-field approximation in the grand canonical ensemble in Ref. [24], but our approach has the advantage of yielding more information about the spatial distribution of channels.

\section{RESULTS}

\section{A. Equilibrium distributions}

We start by studying the equilibrium properties of the system, as defined by our lattice model. We use a meanfield approximation, which will allow us to write explicit expressions for the energy and entropy of the system, from which we can find its free energy. As a simplifying assumption, we assume the existence of at most one cluster. For the energy values we consider, the existence of a single cluster in equilibrium is reasonable and supported by test simulations. Let $f$ be the fraction of channels in the cluster; the other channels are spread throughout the rest of the membrane. Furthermore, let $\phi^{\text {in }}$ be the fraction of open channels within the cluster, and $\phi^{\text {out }}$ the fraction of open channels outside the cluster. The three quantities $f, \phi^{\text {in }}$, and $\phi^{\text {out }}$ are the thermodynamic variables of our model. Our next job is to write the free energy of the system in terms of these variables. Cluster formation and channel gating are then studied by finding the global minimum of the free energy. For example, a cluster is present if $f>0$ in the state of minimum free energy.

The free energy per channel, $F / N$, for a given temperature $T$ can be written as

$$
\frac{F}{N}=\left(e_{\text {int }}+e_{\text {mem }}\right)-T s,
$$

where the entropy per channel, $s$, can be estimated via combinatorial analysis, calculating the number of configurations that channels can assume. The energy per channel is divided into two terms: the interaction among channels, $e_{\text {int }}$, and the interaction of each channel with the membrane, $e_{\text {mem }}$. For each of the configurations devised in the preceding calculation, the interaction among channels can be estimated considering that channels interact only with nearest neighbors. The interaction with the membrane depends on the difference of energies between closed and open states and the work due to the variation on the channel's area in the gating process. In the mean-field approximation, we find (see Appendix A for complete derivation of the results)

$$
\begin{aligned}
s= & k_{B}\left\{\ln \left[\frac{(1-\rho)}{\rho(1-f)\left(1-\phi^{\text {out }}\right)}\right]\right. \\
& +f \ln \left[\frac{\rho(1-f)\left(1-\phi^{\text {out }}\right)}{(1-\rho f)\left(1-\phi^{\text {in }}\right)}\right]+\frac{1}{\rho} \ln \left[\frac{(1-\rho f)}{(1-\rho)}\right] \\
& \left.+\phi^{\text {out }}(1-f) \ln \left[\frac{\left(1-\phi^{\text {out }}\right)}{\phi^{\text {out }}}\right]+\phi^{\text {in }} f \ln \left[\frac{\left(1-\phi^{\text {in }}\right)}{\phi^{\text {in }}}\right]\right\}
\end{aligned}
$$

$e_{\text {int }}=2 f\left[\varepsilon_{c c}+2\left(\varepsilon_{c o}-\varepsilon_{c c}\right) \phi^{\text {in }}+\left(\varepsilon_{c c}-2 \varepsilon_{c o}+\varepsilon_{o o}\right)\left(\phi^{\text {in }}\right)^{2}\right]$;

$$
e_{\mathrm{mem}}=\frac{\left(\Delta G_{0}-\tau \Delta A\right)}{2}\left[2 f \phi^{\text {in }}+2(1-f) \phi^{\text {out }}-1\right] .
$$

Here the parameters $\Delta G_{0}, \Delta A$, and $\tau$ are the difference between the energies of open and closed states, the difference in membrane areas between the open and closed configurations of a channel, and the membrane tension, respectively. The term $\Delta G_{0}$ covers both the energetic cost of membrane deformation and the cost of changing the internal structure of the channel. We used $\Delta G_{0}=50 \quad k_{B} T$ and $\Delta A=20 \mathrm{~nm}^{2}$, following Ref. [25]. For these parameters, a single noninteracting channel has a $50 \%$ opening probability at the tension $\tau=$ $2.5 k_{b} T / \mathrm{nm}^{2}$ [15].

The equilibrium distribution for this system is then given by the values $f_{e q}, \phi_{e q}^{\text {out }}$, and $\phi_{e q}^{\text {in }}$ which minimize the free energy, for given values of $\rho$ and $\tau$ (in the following, the subscript $e q$ 

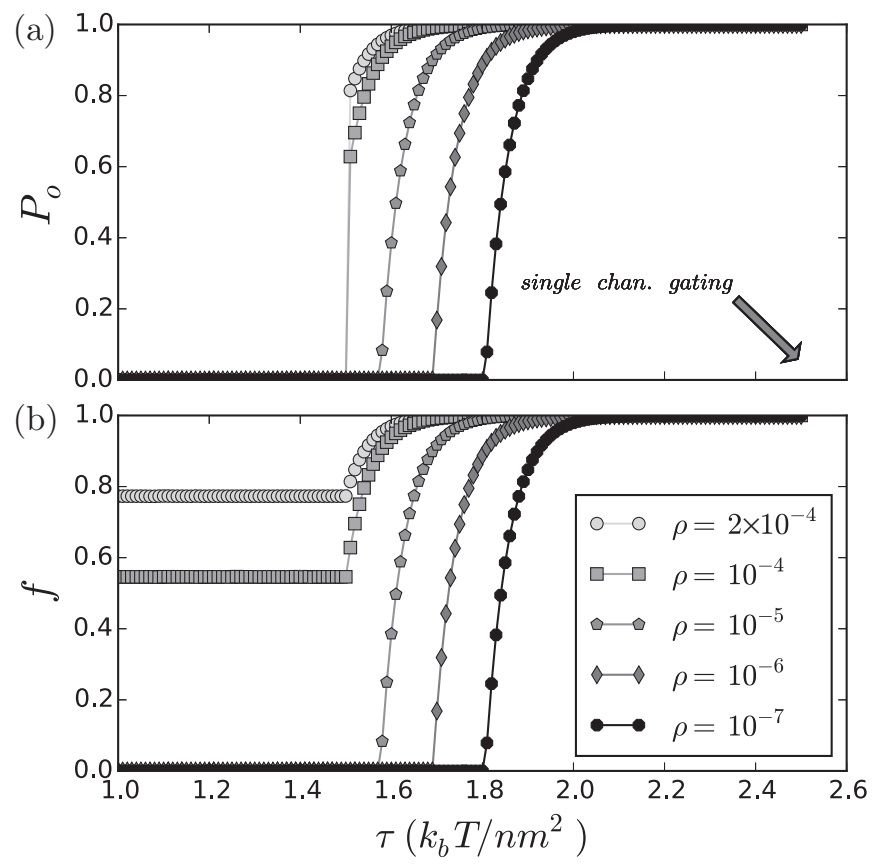

FIG. 2. Fraction of open channels in the system, $P_{o}$ (a), and fraction of channels that belong to the cluster, $f(\mathrm{~b})$, as functions of the membrane tension $\tau$, for different values of $\rho$. Here $\varepsilon_{o o}=-15.0 k_{B} T$, $\varepsilon_{c c}=-5.0 k_{B} T$, and $\varepsilon_{o c}=10.0 k_{B} T$.

will be omitted). The fraction of open channels on the whole lattice is given by $P_{o}=f \phi^{\text {in }}+(1-f) \phi^{\text {out }}$. Figure 2 shows how $f$ and $P_{o}$ vary as functions of the membrane tension, $\tau$, for different values of the density $\rho$.

We see in Fig. 2(a) that the fraction of open channels $P_{o}$ undergoes a transition from nearly zero (all channels closed) to nonzero values as the membrane tension $\tau$ increases. The nature of the transition depends on the channel density $\rho$. For small values of $\rho$, the transition is continuous: $P_{o}$ increases smoothly from zero beyond a critical value of $\tau$. For $\rho$ greater than a critical value $\rho_{c}$, however, the transition is discontinuous, with $P_{o}$ jumping abruptly to a positive value at the critical tension.

The key to explaining this phenomenon is in the corresponding behavior of the cluster fraction $f$, depicted in Fig. 2(b). At low tensions, the channels are closed. Since the force between two closed channels is attractive, they can form a cluster if their density is high enough; this is the case for the two upper curves in Fig. 2(b). Comparing with Fig. 2(a), we see that these correspond to the densities for which the transition in $P_{o}$ is abrupt: if a cluster already exists at low tension, $P_{o}$ has a discontinuous transition. The reason for this comes from the fact that the interaction energy between two open channels is much greater than any other combination of channels, and this becomes more and more so as the tension increases, since high tensions favor the opening of the channels. In equilibrium, if one of the channels in the cluster is open, all the others are open as well, because any mixture of open and closed channels incurs a heavy cost in free energy. So at a critical tension, the whole cluster opens, and since the cluster contains a finite fraction of the channels in the cell, this results in the abrupt jump in $P_{o}$ seen in Fig. 2(a).
For lower channel densities, on the other hand, there is no cluster at lower tensions. As the tension is increased, it eventually becomes favorable for channels to open, and as they do, they will tend to bunch together in a cluster, because of the high open-open interaction energy. But because in this case there was no cluster to start with, the number of open channels will increase gradually as the tension rises, and so will the cluster size. This predicts that the cluster size $f$ and the fraction of open channels $P_{o}$ will undergo a continuous transition and increase in tandem. This is exactly what we see in Fig. 2(a). In both the low- and high-density regimes, the clustering reduces considerably the threshold for channel opening [see Fig. 2(a)], which might have implications for the response of the cell to osmotic shock, as we shall see in the following. These collective phenomena are a direct consequence of the inextricable link between the spatial distribution of channels and their internal gating dynamics.

\section{B. Osmotic shock}

In order to understand the response of the channels to an osmotic shock, we have to go beyond the equilibrium theory and look at their time-dependent activation dynamics. To study the coupled gating and diffusion dynamics, we use a Monte Carlo simulation scheme with two possible actions in each step: (1) with probability $p_{G}$, a randomly chosen channel attempts changing its state (closed or open) or (2) with probability $1-p_{G}$, it attempts to move to one of its four neighboring sites, if it is vacant. The attempts succeed with a probability of acceptance, $A$, according to the criterion $A=e^{-\beta \Delta E}$ if $\Delta E>0$, or $A=1$ if $\Delta E \leqslant 0$, where $\Delta E$ is the change in energy between final and initial configurations of the system following the attempt. Thus, the algorithm is a variation of the Kawasaki dynamics, for which the position updates are local, making it suitable for nonequilibrium simulations of the lattice gas [23]. The probability $p_{G}$ is determined by the ratio of the rates of diffusion and gating: $p_{G}=\lambda_{G} /\left(\lambda_{G}+\lambda_{D}\right)$, where $\lambda_{G}=1 / \Delta t_{G}$ and $\lambda_{D}=1 / \Delta t_{D}$ are the rates of gating and diffusion, given by the experimentally measured characteristic times of gating and diffusion, $\Delta t_{G}$ and $\Delta t_{D}$, respectively. Each Monte Carlo step is given after $N$ random choices of channels to attempt change of state or diffusion, where $N$ is the total number of channels. We relate a Monte Carlo step, $\Delta t_{M C}$, to a real time interval using the weighted average $\Delta t_{M C}=p_{G} \Delta t_{G}+\left(1-p_{G}\right) \Delta t_{D}$. In our simulations, we have used $\Delta t_{G}=4 \mu \mathrm{s}$ and $\Delta t_{D}=208 \mu \mathrm{s}$ [26,27] (see Appendix B), for which we have $\Delta t_{M C} \cong 8 \mu \mathrm{s}$. Since the increase in channel area during the gating process precludes the determination of a single value for the lattice constant, we had to choose it in a range of reasonable biological values. We use $L=400$ and $\rho=0.002$ for lattice size and channel density, respectively, in accordance to typical values for $E$. coli (see Appendix B). In all our simulations, we start the system from an equilibrium situation at low membrane tension $\tau$. We then increase $\tau$ abruptly, mimicking an osmotic shock, and follow the dynamics of the channels using the algorithm described above. The value of $\tau$ is kept fixed throughout the simulation.

It is instructive to compare the time evolution of a hypothetical system of noninteracting channels to that of the 


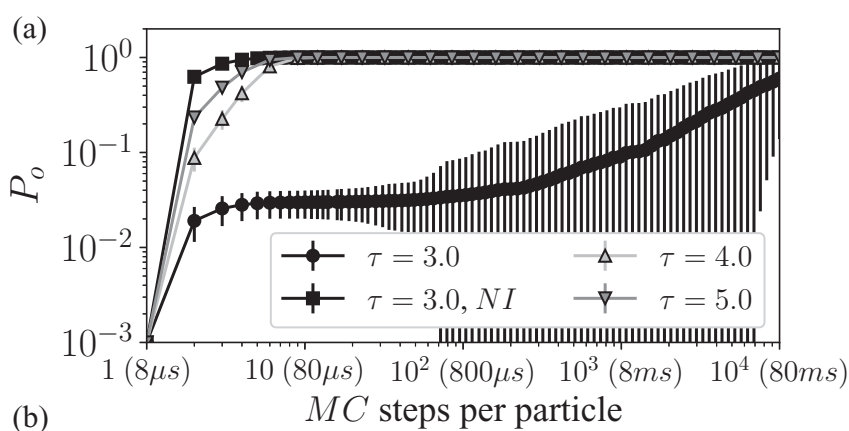

(b)

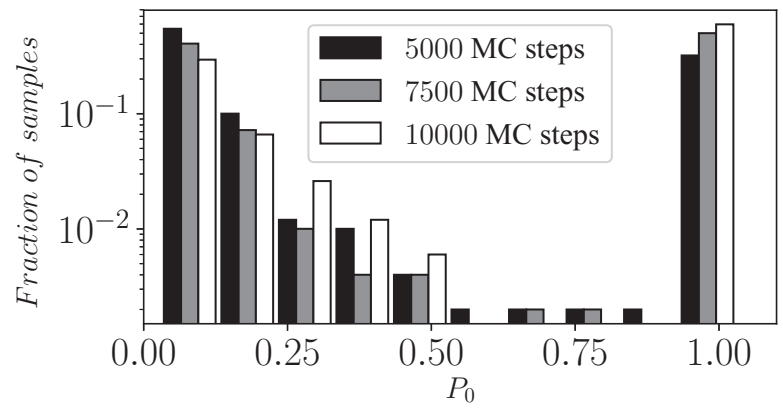

FIG. 3. (a) Average fraction of open channels as a function of simulated time, for different values of $\tau$. Black squares represent the same system without interaction between channels. Real time is shown in parentheses on the $x$ axis. (b) Number of samples with a given fraction of open channels, for three different transients, for the interacting case with $\tau=3.0 k_{b} T / \mathrm{nm}^{2}$. The parameters are $\lambda_{D} / \lambda_{G}=$ $0.02, L=400, \rho=0.002, \varepsilon_{o o}=-15.0 k_{B} T, \varepsilon_{c c}=-5.0 k_{B} T$, and $\varepsilon_{o c}=10.0 k_{B} T$. For each set of parameters, 500 samples were considered, and error bars correspond to one standard deviation.

real system of interacting channels. After approximately 10 MC steps per channel, all the noninteracting channels are open and stay in this state until the end of the simulation [black squares, Fig. 3(a)]. The behavior of the system of interacting channels, in contrast, is governed by two processes acting on vastly different time scales [black circles, Fig. 3(a)]: (1) the fast opening of the isolated channels outside the cluster and (2) the much slower opening of the channels in the cluster. The most striking aspect of the dynamics shown in Fig. 3(a) is the dramatic variability of the opening times of the cluster: in one run of the simulation, the cluster may open in a few microseconds, and in another it may take 100 milliseconds to open. This massive variation is a result of the long-range correlations created by the interactions between channels. The stochastic nature of the cluster is a direct effect of the nontrivial collective behavior of the interactive channels.

The variation is further highlighted by the histogram of the fraction of open channels in a cell some (long) time after the osmotic shock is applied, in 500 independent runs of the Monte Carlo simulation [see Fig. 3(b)]. We see that the distribution is bimodal, with roughly similar numbers of cells with open and closed clusters, even after very long times after the shock. This means that in a population of cells subjected to osmotic shock, there will be massive differences in the response times from one cell to another, even if the cells are genetically identical and even though they feel exactly the same stress. In essence, the collective dynamics that emerged from the channel interactions amplifies stochastic fluctuations at the molecular scale to the "macroscopic," population scale, making them potentially detectable by population assays.

We note that this large variability disappears once the tension becomes strong enough. For $\tau=4.0 k_{b} T / \mathrm{nm}^{2}$ and $5.0 k_{b} T / \mathrm{nm}^{2}$ [respectively, up and down triangles in Fig. 3(a)], both clustered and freely diffusing channels respond very quickly, with all the channels in the system opening after only 10 MC steps.

Finally, we would like to emphasize that the membranes are a crowded environment and that the tight packing of channels within this environment may introduce additional effects on gating. The most important effect appears due to the packing frustration and entropic tension. Packing frustration may lead to a decrease in the tendency to gate due to space limitation created by neighbors. Additionally, entropic tension originated by environmental crowding may have a significant influence on MS channels' conformational change due to volume exclusion [28]. These effects could be considered, as a first approximation, accounting to a different choice of parameters in a simple extension of our model (specifically the energy difference between open and closed states).

\section{CONCLUSION}

Using fluorescence microscopy and Western blot analysis [29], the average number of MscLs in native E. coli cells has been estimated between 300 and 1000 channels. These results are similar to the one obtained with ribosome profiling [30] that measures 360 to 560 channels per cell. These numbers take the channel density close to or above the threshold for cluster formation at low tensions. Patch-clamp experiments complemented with fluorescent and atomic force microscopy show evidence for crowding and collective response of channels in liposomes [31]. Other studies have shown nonhomogeneous distributions of overexpressed MS channels in live bacterial cells [29,32,33]. Although all these studies suggest cluster formation for native channels, the debate around this question is still open. A recent study, through use of photoactivated localization microscopy and single-particle tracking, had shown strong indications that labeling with fluorescent molecules predisposes MscL channels to form clusters [34]. In either case, if there is any form of channel aggregation in bacterial cells, the collective phenomena we describe here may be directly relevant for the osmotic response of bacteria. Furthermore this model can be extended to other types of channels, such as electrically sensitive ion channels, which are also expected to react cooperatively to external stimuli [35].

Possible evidence for the large variability in channel activation predicted by our analysis is the recent observation of very late channel gating activity in $E$. coli cells subjected to osmotic shock [26]: gating was seen as long as $100 \mathrm{~ms}$ after the shock. Since isolated MscL channels are known to gate within a few microseconds after their tension threshold is passed, it is difficult to explain this observation if the channels do not interact. This is naturally explained by the variability of channel activation, however: Fig. 3 shows that a cluster could take a time of the order of $100 \mathrm{~ms}$ to open. Another recent work [36] also highlights the large cell-to-cell variability of the downshock responses. Furthermore this work shows 
a very slow cell volume recovery, which may also indicate cooperative activity of channels.

Calculations based on the ionic flux through a single open channel suggest that as few as five to 10 channels would be enough to protect a cell [26]. This contrasts with the recent measurements of $\mathrm{MscL}$ numbers on native cells, which indicate numbers of channels up to two orders of magnitude greater than this estimate. With so many channels in a native $E$. coli cell, simultaneous opening of all channels would lead to a drastic release of intracellular material, as well as depolarization of the membrane potential [26], with potentially fatal consequences for the cell. Hence this high expression level of channels is still a mystery. As seen in Fig. 3, the presence of the cluster significantly delays the opening of the whole system of channels, compared with the noninteracting case, for a shock with smaller membrane tension. Thus, clustering could provide a mean to self-regulate the simultaneous opening of a large number of channels, in order to restore the osmotic equilibrium of the cell and function as a channel reservoir if more of them are needed in case of a severe shock. This is an admittedly speculative, but plausible, fitness advantage for the large numbers of channels found in E. coli.

\section{ACKNOWLEDGMENTS}

It is a pleasure to thank I. Booth, H. Galbiati, and S. Miller for important discussions. This work was supported by the European Union Seventh Framework Programme [FP7/20072013] (NICHE; Grant Agreement No. 289384).

\section{APPENDIX A: DERIVATION FOR THE FREE ENERGY EXPRESSION}

Consider a square lattice of size $L$, in which each site can be empty or occupied by a channel, with a total of $N$ indistinguishable channels on the lattice. These channels are either grouped in a cluster of size $n_{c}$ or scattered on the lattice and can assume two possible states: open or closed. Let us assume that there are $o$ open channels and $c$ closed channels on this lattice and also assume that $o_{\text {in }}$ of these open channels belong to the cluster and $o_{\text {out }}$ are scattered (in a same sense, there are $c_{\text {in }}$ closed channels in the cluster and $c_{\text {out }}$ closed channels scattered, so that the relations $o=o_{\text {in }}+o_{\text {out }}$ and $c=c_{\text {in }}+c_{\text {out }}$ are valid).

The total number of configurations for this system will be the product of the number of configurations of the cluster and the number of configurations of the area outside the cluster. The number of configurations for the cluster (disregarding permutations between indistinguishable channels) is given by

$$
\frac{n_{c} !}{o_{\text {in }} ! c_{\mathrm{in}} !} \text {. }
$$

The number of configurations of the area outside the cluster considers the permutation of the sites $\left(\left(L^{2}-n_{c}\right)\right.$ !) ignoring permutations between channels and permutation between empty sites. Thus we have

$$
\frac{\left(L^{2}-n_{c}\right) !}{o_{\text {out }} ! c_{\text {out }} !\left(L^{2}-N\right) !}
$$

Using the relations

$$
\begin{aligned}
o-o_{\text {in }} & =o_{\text {out }}, \\
c-c_{\text {in }} & =N-n_{c}-o_{\text {out }}, \\
c_{\text {in }} & =n_{c}-o_{\text {in }},
\end{aligned}
$$

we obtain for the product of Eqs. (A1) and (A2):

$$
\frac{n_{c} !\left(L^{2}-n_{c}\right) !}{o_{\text {in }} !\left(n_{c}-o_{\text {in }}\right) ! o_{\text {out }} !\left(N-n_{c}-o_{\text {out }}\right) !\left(L^{2}-N\right) !}
$$

or even in a more elegant form:

$$
\frac{1}{\left(N-n_{c}\right) !} \frac{\left(L^{2}-n_{c}\right) !}{\left(L^{2}-N\right) !}\left(\begin{array}{c}
N-n_{c} \\
o_{\text {out }}
\end{array}\right)\left(\begin{array}{c}
n_{c} \\
o_{\text {in }}
\end{array}\right) .
$$

With the total number of configurations of indistinguishable channels given by (A3), we can calculate the entropy for the system:

$$
S=k_{B} \log \left[\frac{n_{c} !\left(L^{2}-n_{c}\right) !}{o_{\text {in }} !\left(n_{c}-o_{\text {in }}\right) ! o_{\text {out }} !\left(N-n_{c}-o_{\text {out }}\right) !\left(L^{2}-N\right) !}\right],
$$

which gives (using Stirling's formula)

$$
\begin{aligned}
S= & k_{B}\left\{N \log \left[\frac{\left(L^{2}-N\right)}{\left(N-n_{c}-o_{\text {out }}\right)}\right]\right. \\
& +n_{c} \log \left[\frac{n_{c}\left(N-n_{c}-o_{\text {out }}\right)}{\left(L^{2}-n_{c}\right)\left(n_{c}-o_{\text {in }}\right)}\right] \\
& +L^{2} \log \left[\frac{\left(L^{2}-n_{c}\right)}{\left(L^{2}-N\right)}\right] \\
& +o_{\text {out }} \log \left[\frac{\left(N-n_{c}-o_{\text {out }}\right)}{o_{\text {out }}}\right] \\
& \left.+o_{\text {in }} \log \left[\frac{\left(n_{c}-o_{\text {in }}\right)}{o_{\text {in }}}\right]\right\} .
\end{aligned}
$$

Defining the quantities

$$
\rho=\frac{N}{L^{2}}, \quad f=\frac{n_{c}}{N}, \quad \phi^{\text {out }}=\frac{o_{\text {out }}}{N-n_{c}}, \quad \phi^{\text {in }}=\frac{o_{\text {in }}}{n_{c}},
$$

we have

$$
\begin{aligned}
S= & k_{B} N\left\{\log \left[\frac{(1-\rho)}{\rho(1-f)\left(1-\phi^{\text {out }}\right)}\right]\right. \\
& +f \log \left[\frac{\rho(1-f)\left(1-\phi^{\text {out }}\right)}{(1-\rho f)\left(1-\phi^{\text {in }}\right)}\right] \\
& +\frac{1}{\rho} \log \left[\frac{(1-\rho f)}{(1-\rho)}\right]+\phi^{\text {out }}(1-f) \log \left[\frac{\left(1-\phi^{\text {out }}\right)}{\phi^{\text {out }}}\right] \\
& \left.+\phi^{\text {in }} f \log \left[\frac{\left(1-\phi^{\text {in }}\right)}{\phi^{\text {in }}}\right]\right\} .
\end{aligned}
$$

To calculate the energy of the channels inside the cluster, consider $P_{m}$ as the probability that $m$ of the four neighbors of a chosen node are open. This probability can be estimated by

$$
\begin{aligned}
P_{m} & \simeq\left(\begin{array}{c}
4 \\
m
\end{array}\right)\left(\frac{o_{\text {in }}}{n_{c}}\right)^{m}\left(1-\frac{o_{\text {in }}}{n_{c}}\right)^{4-m} \\
& =\left(\begin{array}{c}
4 \\
m
\end{array}\right)\left(\phi^{\text {in }}\right)^{m}\left(1-\phi^{\text {in }}\right)^{4-m} .
\end{aligned}
$$


If $\varepsilon_{o o}, \varepsilon_{c o}$, and $\varepsilon_{c c}$ are the interaction energies between two open channels, one closed channel and one open channel, and two closed channels, respectively, then the energy that accounts for the interactions inside the cluster can be estimated by

$$
\begin{aligned}
E_{\text {int }}= & \frac{o_{\text {in }}}{2}\left[\left(4 \varepsilon_{c o}\right) P_{0}+\left(3 \varepsilon_{c o}+\varepsilon_{o o}\right) P_{1}+\left(2 \varepsilon_{c o}+2 \varepsilon_{o o}\right) P_{2}\right. \\
& \left.+\left(\varepsilon_{c o}+3 \varepsilon_{o o}\right) P_{3}+\left(4 \varepsilon_{o o}\right) P_{4}\right] \\
& +\frac{\left(n_{c}-o_{\text {in }}\right)}{2}\left[\left(4 \varepsilon_{c c}\right) P_{0}+\left(3 \varepsilon_{c c}+\varepsilon_{c o}\right) P_{1}\right. \\
& \left.+\left(2 \varepsilon_{c c}+2 \varepsilon_{c o}\right) P_{2}+\left(\varepsilon_{c c}+3 \varepsilon_{c o}\right) P_{3}+\left(4 \varepsilon_{c o}\right) P_{4}\right],
\end{aligned}
$$

which can be further simplified to

$$
E_{\mathrm{int}}=2 N f\left[\varepsilon_{c c}+2\left(\varepsilon_{c o}-\varepsilon_{c c}\right) \phi^{\mathrm{in}}+\left(\varepsilon_{c c}-2 \varepsilon_{c o}+\varepsilon_{o o}\right)\left(\phi^{\mathrm{in}}\right)^{2}\right] \text {. }
$$

The region outside the cluster is only sparsely populated by channels, so that the chance of a close encounter between two channels is very small. Given that, we disregard any interaction energy between channels outside the cluster.

For all the channel on the lattice it is also needed to consider the conformation energy, as a function of the membrane tension, or, in other words, how the channels sense variations of this tension. Considering all channels on the lattice, we have

$$
\begin{aligned}
E_{\text {mem }}= & \left(o_{\text {in }}+o_{\text {out }}\right) \frac{\left(\Delta G_{0}-\tau \Delta A\right)}{2} \\
& -\left(N-o_{\text {in }}-o_{\text {out }}\right) \frac{\left(\Delta G_{0}-\tau \Delta A\right)}{2} \\
= & \frac{\left(\Delta G_{0}-\tau \Delta A\right)}{2}\left(2 o_{\text {in }}+2 o_{\text {out }}-N\right) \\
= & N \frac{\left(\Delta G_{0}-\tau \Delta A\right)}{2}\left[2 f \phi^{\text {in }}+2(1-f) \phi^{\text {out }}-1\right],
\end{aligned}
$$

where $\Delta G_{0}$ is the difference between the energies of open and closed states, $\Delta A$ is the deformation area of the channels and $\tau$ is the membrane tension. We then have for the total energy of the system, $E=E_{\text {int }}+E_{\text {mem }}$ :

$$
\begin{aligned}
E=N & \left\{2 f \left[\varepsilon_{c c}+2\left(\varepsilon_{c o}-\varepsilon_{c c}\right) \phi^{\text {in }}\right.\right. \\
& \left.+\left(\varepsilon_{c c}-2 \varepsilon_{c o}+\varepsilon_{o o}\right)\left(\phi^{\text {in }}\right)^{2}\right] \\
& \left.+\frac{\left(\Delta G_{0}-\tau \Delta A\right)}{2}\left[2 f \phi^{\text {in }}+2(1-f) \phi^{\text {out }}-1\right]\right\} .
\end{aligned}
$$

Finally, we have for the system's free energy:

$$
\begin{aligned}
F= & E-T S \\
= & N\left\{2 f \left[\varepsilon_{c c}+2\left(\varepsilon_{c o}-\varepsilon_{c c}\right) \phi^{\text {in }}\right.\right. \\
& \left.+\left(\varepsilon_{c c}-2 \varepsilon_{c o}+\varepsilon_{o o}\right)\left(\phi^{\text {in }}\right)^{2}\right] \\
& \left.+\frac{\left(\Delta G_{0}-\tau \Delta A\right)}{2}\left[2 f \phi^{\text {in }}+2(1-f) \phi^{\text {out }}-1\right]\right\} \\
& -N k_{B} T\left\{\log \left[\frac{(1-\rho)}{\rho(1-f)\left(1-\phi^{\text {out }}\right)}\right]\right.
\end{aligned}
$$

$$
\begin{aligned}
& +f \log \left[\frac{\rho(1-f)\left(1-\phi^{\text {out }}\right)}{(1-\rho f)\left(1-\phi^{\text {in }}\right)}\right] \\
& +\frac{1}{\rho} \log \left[\frac{(1-\rho f)}{(1-\rho)}\right] \\
& +\phi^{\text {out }}(1-f) \log \left[\frac{\left(1-\phi^{\text {out }}\right)}{\phi^{\text {out }}}\right] \\
& \left.+\phi^{\text {in }} f \log \left[\frac{\left(1-\phi^{\text {in }}\right)}{\phi^{\text {in }}}\right]\right\} .
\end{aligned}
$$

\section{APPENDIX B: LATTICE SIZE, DENSITY AND CHARACTERISTIC TIMES FOR GATING AND DIFFUSION}

As a simplifying approach to study the gating dynamics, we consider a membrane which is solely populated by MscL. To obtain the total number of lattice sites $N_{s}$, we divide the total membrane area of E. coli, $\approx 6 \times 10^{-12} \mathrm{~m}^{2}$ [37], by the area of a single channel. As the area occupied by a single channel increases during the gating process, it is not possible to determine a single value for this parameter. Considering the radius of the closed channel, $2.5 \mathrm{~nm}$, this gives a total number of lattice sites equal to $N_{s}=305577$, and hence, for a regular square lattice, we have a lattice size $L=\sqrt{N_{s}} \approx 553$. If on the other hand we consider the radius of the open channel, $3.5 \mathrm{~nm}$, we have $N_{s}=155907$, and thus $L \approx 395$. In the simulations, we use $L=400$, as it is still in the biologically relevant interval, and because small variations in this parameter will not change qualitatively the results.

As discussed in Ref. [26], previous studies suggest that the channels gate $\sim 3-5 \mu$ s after tension threshold is reached. Thus, we set

$$
\Delta t_{G}=4 \mu \mathrm{s}
$$

For the diffusion coefficient of MscL we choose $D=$ $0.059 \mathrm{~nm}^{2} / \mu \mathrm{s}$, a value close to the one obtained experimentally in Ref. [34]. Considering diffusion in a bidimensional circle of radius $2 r$, where $r=3.5 \mathrm{~nm}$ is the radius of a single open channel (approximately to the size of one lattice cell), we can obtain an estimate of the diffusion time using $\sigma^{2}=4 D t$, with $\sigma \approx 2 r$. Thus,

$$
t=\frac{\sigma^{2}}{4 D}=\frac{49}{4 \times 0.059} \approx 208 \mu \mathrm{s} .
$$

With this, we set

$$
\Delta t_{D}=208 \mu \mathrm{s} .
$$

Finally, considering the quantification of MscL in E. coli done in Ref. [29], the number of channels is estimated in the range $300-1000$. We use the value $N=320$, which gives a density $\rho=320 /(400)^{2}=0.002$. For the interaction energies used before, $\varepsilon_{o o}=-15 k_{B} T, \varepsilon_{c c}=-5 k_{B} T$, and $\varepsilon_{o c}=10 k_{B} T$, this density is above the threshold for cluster formation in the lattice gas model. 
[1] B. Poolman, J. J. Spitzer, and J. M. Wood, Biochim. Biophys. Acta 1666, 88 (2004).

[2] J. M. Wood, E. Bremer, L. N. Csonka, R. Kraemer, B. Poolman, T. van der Heide, and L. T. Smith, Comp. Biochem. Physiol. A 130, 437 (2001).

[3] M. Roberts, Front. Biosci. 5, d796 (2000).

[4] K. Wegmann, FEMS Microbiol. Lett. 39, 37 (1986).

[5] A. Brown, K. Mackenzie, and K. Singh, FEMS Microbiol. Lett. 39, 31 (1986).

[6] A. Blomberg, Electrophoresis 18, 1429 (1997).

[7] B. Martinac and M. Buechner, Proc. Natl. Acad. Sci. USA 84, 2297 (1987).

[8] S. Sukharev, B. Martinac, V. Arshavsky, and C. Kung, Biophys. J. 65, 177 (1993).

[9] C. Cui, D. Smith, and J. Adler, J. Membr. Biol. 144, 31 (1995).

[10] N. Levina, S. Tötemeyer, and N. Stokes, EMBO J. 18, 1730 (1999).

[11] C. Kung, Nature (London) 436, 647 (2005).

[12] I. R. Booth, M. D. Edwards, S. Black, U. Schumann, and S. Miller, Nature Rev. Microbiol. 5, 431 (2007).

[13] C. D. Pivetti, M.-R. Yen, S. Miller, W. Busch, Y.-h. Tseng, I. R. Booth, H. Saier, and M. H. Saier, Microbiol. Mol. Biol. Rev. 67, 66 (2003).

[14] R. Phillips, T. Ursell, P. Wiggins, and P. Sens, Nature (London) 459, 379 (2009).

[15] T. Ursell, K. C. Huang, E. Peterson, and R. Phillips, PLoS Comput. Biol. 3, e81 (2007).

[16] A. V. Botelho, T. Huber, T. P. Sakmar, and M. F. Brown, Biophys. J. 91, 4464 (2006).

[17] M. L. Skoge, R. G. Endres, and N. S. Wingreen, Biophys. J. 90, 4317 (2006).

[18] K. Guseva, M. Thiel, I. Booth, S. Miller, C. Grebogi, and A. de Moura, Phys. Rev. E 83, 020901 (2011).

[19] K. Guseva, Formation and Cooperative Behaviour of Protein Complexes on the Cell Membrane, Springer Theses (Springer, Berlin, 2011).

[20] A. F. Batiza, M. M.-C. Kuo, K. Yoshimura, and C. Kung, Proc. Natl. Acad. Sci. USA 99, 5643 (2002).
[21] A. Kloda, E. Petrov, G. R. Meyer, T. Nguyen, A. C. Hurst, L. Hool, and B. Martinac, Intl. J. Biochem. Cell Biol. 40, 164 (2008).

[22] G. Chang, R. H. Spencer, A. T. Lee, M. T. Barclay, and D. C. Rees, Science 282, 2220 (1998).

[23] E. Newman and G. Barkema, Monte Carlo Methods in Statistical Physics (Clarendon Press, Oxford, 1999).

[24] J. Sivardière and J. Lajzerowicz, Phys. Rev. A 11, 2090 (1975).

[25] C.-S. Chiang, A. Anishkin, and S. Sukharev, Biophys. J. 86, 2846 (2004).

[26] I. R. Booth, Curr. Opin. Microbiol. 18, 16 (2014).

[27] M. Kumar, M. S. Mommer, and V. Sourjik, Biophys. J. 98, 552 (2010).

[28] M. Lindén, P. Sens, and R. Phillips, PLoS Comput. Biol. 8, e1002431 (2012).

[29] M. Bialecka-Fornal, H. J. Lee, H. A. DeBerg, C. S. Gandhi, and R. Phillips, PloS One 7, e33077 (2012).

[30] G.-W. Li, D. Burkhardt, C. Gross, and J. S. Weissman, Cell 157, 624 (2014).

[31] S. L. Grage, A. M. Keleshian, T. Turdzeladze, A. R. Battle, W. C. Tay, R. P. May, S. A. Holt, S. A. Contera, M. Haertlein, M. Moulin, P. Pal, P. R. Rohde, V. T. Forsyth, A. Watts, K. C. Huang, A. S. Ulrich, and B. Martinac, Biophys. J. 100, 1252 (2011).

[32] P. G. Wahome, A. E. Cowan, B. Setlow, and P. Setlow, Arch. Microbiol. 191, 403 (2009).

[33] C. Norman, Z.-W. Liu, P. Rigby, A. Raso, Y. Petrov, and B. Martinac, Eur. Biophys. J. 34, 396 (2005).

[34] J. van den Berg, H. Galbiati, A. Rasmussen, S. Miller, and B. Poolman, Sci. Rep. 6, 32709 (2016).

[35] J. Cervera, J. A. Manzanares, and S. Mafe, J. R. Soc. Interface 11, 20140099 (2014).

[36] R. Buda, Y. Liu, J. Yang, S. Hegde, K. Stevenson, F. Bai, and T. Pilizota, Proc. Natl. Acad. Sci. USA 113, E5838 (2016).

[37] R. Phillips, J. Kondev, J. Theriot, and H. Garcia, Physical Biology of the Cell (Taylor \& Francis Group, Philadelphia, 2012). 